\title{
An analysis of translocation regimes for the endangered puaiohi Myadestes palmeri
}

\author{
Jean Fantle-Lepczyk ${ }^{1,4, *}$, Lisa H. Crampton ${ }^{2}$, Andrew Taylor ${ }^{1}$, David C. Duffy ${ }^{3}$, \\ Sheila Conant ${ }^{1}$
}

\author{
${ }^{1}$ Department of Biology, University of Hawai'i at Mānoa, Honolulu, HI 96822, USA \\ ${ }^{2}$ Kaua'i Forest Birds Recovery Project, Hawai'i Division of Forestry and Wildlife and University of Hawai'i at Mānoa, \\ Hanapepe, HI 96716, USA \\ ${ }^{3}$ Pacific Cooperative Studies Unit, Department of Botany, University of Hawai'i at Mānoa, Honolulu, HI 96822, USA \\ ${ }^{4}$ Present address: Auburn University, School of Forestry \& Wildlife Sciences, Auburn, AL 36830, USA
}

\begin{abstract}
The ongoing and often synergistic effects of habitat loss, invasive species, and climate change pose challenges for conservation and management as widespread species become greatly reduced, sometimes to a single small population. To address this problem, conservation biologists must consider using approaches like translocation to create new populations, reducing the probability of extinction by splitting a population into 2 or more populations in geographically distinct locales. The puaiohi Myadestes palmeri, an endangered Hawaiian forest bird, has a small population size (494; $95 \%$ CI: 414-580) and restricted range $\left(\sim 40 \mathrm{~km}^{2}\right)$. One recovery plan objective involves translocating birds to higher elevation Hawaiian Islands. To evaluate translocation scenarios, we built upon previously developed population viability analysis models and considered how translocation regimes (initial harvest/population, number harvested/supplemented per event, harvest/supplementation interval, and length of harvest/supplementation) would affect both original and new populations. Furthermore, we modeled the puaiohi release population under 3 different conditions: a stable population, a predator-controlled environment, and a habitat improved in terms of resource availability. Our results indicated that while translocation offers hope of increasing puaiohi population size and decreasing extinction risk, success will depend on conditions at the release site. Furthermore, harvest and rearing of eggs to the juvenile stage or reestablishment of a captive breeding program may be necessary to provide enough birds to translocate, as the current wild population may not be productive enough to sustain levels of harvest necessary to successfully establish a new population.
\end{abstract}

KEY WORDS: Captive breeding $\cdot$ Captive rearing $\cdot$ Hawaiian forest birds $\cdot$ Population viability analysis $\cdot$ PVA $\cdot$ Small Kaua'i thrush $\cdot$ Vortex

\section{INTRODUCTION}

The planet's ongoing extinction crisis poses challenges for species conservation and management as biologists are forced to negotiate both long-standing problems, such as habitat loss and invasive species, and more recent problems like climate change. Species that were once widespread have been greatly

${ }^{*}$ Corresponding author: fantle@auburn.edu reduced, sometimes resulting in only a single small population. For instance, California condor Gymnogyps californianus and whooping crane Grus americana were each reduced to single small populations as a result of overexploitation, pollution, and habitat loss (Meine \& Archibald 1996, Meretsky et al. 2000). When species exist as only a single small population, they face a wide variety of challenges, including

(C) The authors 2020. Open Access under Creative Commons by Attribution Licence. Use, distribution and reproduction are unrestricted. Authors and original publication must be credited. 
increased likelihood of extirpation due to stochastic events and inbreeding depression (Shaffer 1981, Gilpin \& Soulé 1986, Lacy 2000). Because of the ongoing and often synergistic effects of habitat loss, invasive species, and climate change, managers need to consider more intrusive and labor-intensive approaches to bring species back from the brink of extinction. One such approach is to create new populations via reintroduction into habitat from which the species was extirpated or translocation into novel but biologically appropriate geographic areas (Griffith et al. 1989). The goal of these approaches is the creation of additional populations, thereby reducing the probability of extinction by spreading risk.

While novel and emergent conservation approaches are relevant throughout the world, nowhere are they more needed than the Hawaiian Islands, home to the largest number of threatened and endangered species in the USA (Wilcove et al. 1998), and one of the most imperiled avifaunas in the world (Scott et al. 2001, Pratt 2009). Amongst the species with a single population left in Hawaii, one in urgent need of reduction in risk of extinction is the puaiohi Myadestes palmeri. The puaiohi, or small Kaua'i thrush, is endemic to Kaua'i and is the only remaining native frugivore and only remaining member of Turdidae on the island. Over the past $30 \mathrm{yr}, 5$ other species disappeared from the island following Hurricanes 'Iwa (1982) and 'Iniki (1992) (Conant et al. 1998). Though some of these species were rare in the early 20th century (Perkins 1903), most were more common than the puaiohi. In fact, its congener, the kāma'o, was once the most common forest bird on Kaua'i (Perkins 1903, Scott et al. 1986), yet the puaiohi has persisted while the kāma'o is extinct, perhaps due to the puaiohi's habit of nesting in sheltered ravines. The puaiohi has a population of 494 (95\% CI: 414-580) and is currently restricted to a remnant of the Alaka'i Wilderness Preserve at 1050 to $1300 \mathrm{~m}$, where $75 \%$ of its breeding population occurs in just $10 \mathrm{~km}^{2}$ of forest (USFWS 2006, Crampton et al. 2017).

Many issues contribute to the vulnerability of the puaiohi population, including drought, hurricanes, invasive predators (e.g. rats Rattus spp. and cats Felis catus), disease (particularly infection with avian malaria Plasmodium relictum), and habitat degradation due to feral livestock (pigs and goats) and invasive plants (Kepler \& Kepler 1983, Snetsinger et al. 2005, USFWS 2006, Woodworth \& Pratt 2009). However, recent work suggests that traditional conservation efforts to control introduced predators, remove invasive species, and restore native forest may be insufficient to protect many Hawaiian forest birds, including the puaiohi, from further decline as climate change affects the distribution of avian malaria within the Islands (Benning et al. 2002, Kilpatrick 2006, Fortini et al. 2015). Though infection with malaria may not affect puaiohi survival as negatively as it does other species (VanderWerf et al. 2014), given the puaiohi's absence from malaria-infected lower elevations, malaria is likely to have at least some impact on the population. Thus, unless managers explore alternative, long-term solutions to managing the species, they risk species' range collapse and extinction (Fortini et al. 2015).

Concerns about puaiohi's limited range and small population led to the establishment of a captive-bred population in 1996. From 1999 to 2012, 222 birds were released into the Alaka'i (Switzer et al. 2013). However, due to the relatively low recruitment and survival of released birds in later years of the program and signs of abnormalities in captive bred chicks, the program was discontinued and the last of the captive flock was released in 2017 (Switzer et al. 2013, VanderWerf et al. 2014, Hawai'i Department of Land and Natural Resources 2016).

As the climate warms, increasing the threat of malaria, and invasive species negatively impact both the species and the habitat upon which they rely, the persistence of puaiohi in their remaining forest becomes more precarious. Furthermore, given the limitation of nest sites and perhaps other resources within current habitat, puaiohi may be at their carrying capacity (Snetsinger et al. 2005). In addition, as evidenced after the 1982 and 1992 hurricanes 'Iwa and 'Iniki, in which 5 of the 6 endangered species in the Alaka'i Swamp went extinct, small populations on Kaua'i are extremely vulnerable to hurricanes (Conant et al. 1998). One way to increase overall population size and reduce the dangers of catastrophic events would be to establish one or more additional populations that are geographically separated from the current one, as was accomplished with the Nihoa millerbird Acrocephalus familiaris kingi (Freifeld et al. 2016) and Seychelles warbler $A$. sechellensis (Komdeur 1997). These factors have led to an increasing interest in translocation as an option for species recovery. Thus, while continued monitoring and management of puaiohi within their current range remains a primary concern, one of the current recovery objectives for puaiohi is to investigate the feasibility of translocating birds to higher elevation Hawaiian Islands such as Maui and Hawai'i (Hawai'i Forest Bird Conservation Forum, September 24-25, 2015). 
To evaluate future conservation approaches for the species, we modeled potential outcomes of translocating puaiohi to establish another population and identified sustainable levels of effort and support necessary to successfully create the new population, while minimizing effects on the current population. Previous modeling efforts in Hawai'i (Conant \& Morin 2001) led to the successful translocation of the Nihoa millerbird from Nihoa to Laysan, an atoll from which millerbirds had been absent for approximately 100 yr (Ely \& Clapp 1973). As with millerbirds, the objective of our modeling efforts is to evaluate whether translocation offers a feasible tool for increasing puaiohi populations, and if so, identify how probability of success can be maximized.

\section{METHODS}

We calculated model parameters from data collected from 2005 to 2011 at 4 sites (Kawaikōì, Koaie, Mohihi, and Halepa'akai) in the Alaka'i Wilderness Preserve on the island of Kaua'i, Hawai'i. These sites ranged in elevation from 1123 to $1303 \mathrm{~m}$ above sea level in native wet and mesic forests dominated by 'ōhi'a Metrosideros polymorpha, koa Acacia koa, ōlapa Cheirodendron trigynum, lapalapa C. platyphyllum, 'ōhi'a ha Syzygium sandwicensis, kāwa'u Ilex anomala, and kōlea Myrsine lessertiana, with a diverse understory of native plants including 'ōhelo Vaccinium calycinum and kanawao Broussaisia arguta (USFWS 2006).

To evaluate translocation scenarios, we constructed population viability analysis (PVA) models using Vortex 10 (Lacy \& Pollak 2014), which simulates stochastic demographic and environmental processes. Within Vortex we built upon previously developed models (Fantle-Lepczyk et al. 2018) using all available information on puaiohi. In addition to running a baseline control model in which no translocation occurred, we considered how translocation regimes affected both the original and the new populations. Specifically, we modeled the initial number harvested from the source population (initial population; 10 or 20 birds), the number harvested from the source and supplemented to the new population (number harvested/supplemented per event; 5, 10, or 20 birds), how frequently birds were harvested and supplemented (harvest/supplementation interval; every 1, 2, or $5 \mathrm{yr}$ ) and how long harvest and supplementation lasted (length of harvest/supplementation; 5 or $10 \mathrm{yr}$ ). We considered 3 different sources of birds to translocate: a declin- ing wild population ( $r=-0.267$; Fantle-Lepczyk et al. 2018), a stable wild population (Crampton et al. 2017), and a captive-bred source with no impact on the wild population. We considered the 2 different wild source population dynamics because although baseline models predict a population declining to extinction, scientists have assumed puaiohi populations to have been stable for the last $40 \mathrm{yr}$ (USFWS 2006, Crampton et al. 2017). Modeling under both conditions allowed us to examine the possible range of dynamics currently affecting the wild population. We also considered 2 wild sources of translocatable birds (directly translocated juvenile birds and eggs collected to be reared and released as juveniles), in addition to the captive bred birds. Further, we modeled the translocated population under 3 different release conditions: the same as those extrapolated for a wild stable population, those in a predator-controlled environment, and those in a habitat improved in terms of resource availability.

Each model was simulated 1000 times (OwenSmith 2007) over 25 yr, a relatively short time frame. Although our primary goal is long-term preservation of the species, urgent short-term needs currently drive the recovery program. Furthermore, longer time spans can produce higher extinction probabilities (Akçakaya et al. 1999), more uncertain events (Akçakaya 2005), and propagate errors (Beissinger \& Westphal 1998). Thus, we selected 25 yr as it allowed us to minimize the effects of uncertainties or errors in our parameter estimates, while exploring and testing the immediate effects of management strategies. Vortex's standard output provided growth rate (stochastic $r$ ), probability of extinction, average population size at $25 \mathrm{yr}$, and mean time to extinction. For our modeling purposes, extinction occurred when only one sex remained. We developed our demographic input parameters from a variety of sources, including previously published information, original data, and expert discussion. Baseline models were previously published in Fantle-Lepczyk et al. (2018).

\subsection{Harvest and supplementation}

To determine sustainable translocation regimes and their impacts on the existing puaiohi population, we modeled all combinations of variations of the initial harvest/starting population of the translocated population, the number of birds harvested/ supplemented at each supplementation interval, 
length of harvest/supplementation interval, and the length of harvest/supplementation ( $\mathrm{n}=36$, Table 1). Specifically, from a source population of 500 birds, we modeled the effects of initially removing 10 or 20 birds, followed by regular harvests of either 5, 10, or 20 birds. Harvests occurred every 1, 2, or 5 yr during either a 5 or 10 yr period. For evaluation purposes, we calculated 2 additional measures from the latter 3 variables: number of harvests/supplements and total number of birds harvested/supplemented. We assumed 90\% survival immediately after translocation, as previous releases of captive-bred puaiohi found a $91 \% 30$ d post-release survival (Tweed et al. 2006) for primarily juvenile birds. Subsequent releases saw decreasing survival (Switzer et al. 2013), though it is possible this was due to the release of older birds or the declining fitness of the captive population. We chose to translocate only juvenile birds, as these exhibited the highest survival and integration into the existing population in previous releases of captive-bred puaiohi (Tweed et al. 2006, Switzer et al. 2013, VanderWerf et al. 2014).

We also modeled collecting eggs and hatching and rearing the resulting nestlings until releasable. Presumably, given the high re-nesting rate of puaiohi (Snetsinger et al. 2005), the removal of eggs early in incubation would have little impact on the population overall. We assumed we would need to catch $25 \%$ more birds or harvest $25 \%$ more eggs to allow for unfit birds, infertile eggs, and nestling mortality. Thus, to rear and release 5 juvenile puaiohi, we assumed we would need to collect 7 eggs (4 nest attempts); for 10 juveniles, 13 eggs ( 7 nest attempts); and for 20 juveniles, 25 eggs (13 nest attempts); each from as many different pairs as possible. To model the effect of egg collection, we adjusted the distribution of successful nesting attempts to reflect the nest attempts lost to harvest.

Table 1. Puaiohi population variables evaluated across a range of scenarios

\begin{tabular}{|ll|}
\hline Variable & Conditions \\
\hline Source population & $\begin{array}{l}\text { Wild-declining or stable baseline } \\
\text { Captive-reared - declining or stable baseline } \\
\text { Captive-bred }\end{array}$ \\
Initial population size & 10 or 20 individuals \\
No. supplemented & 5,10, or 20 individuals \\
Supplement interval & Every 1, 2, or 5 yr \\
No. years supplemented & 5 or 10 \\
Release conditions & Stable mortality, predator-controlled, or im- \\
& proved habitat \\
\hline
\end{tabular}

\subsection{Translocated population demographics}

We considered 3 different sets of conditions following release. Under each condition we assumed the release site would be an improvement over current conditions, which at best produce a small and stable, though potentially declining, population. The first of the 3 release conditions reflects a stable population, as extrapolated for the wild stable population (Fantle-Lepczyk et al. 2018). Second, we modeled release in a predator-controlled area in which survival and nest success increase due to release from predator impacts. Finally, we modeled release into an area with comparatively improved habitat, in which increased food sources and nesting sites and some degree of predator control would allow for a greater increase in both survival and reproductive output.

With our models exhibiting stable mortality at release sites, we explored the effect of releasing puaiohi into an area managed to increase puaiohi survival at all life stages, while holding steady all other demographics. To model eliminating the effects of malaria, predation, or any other mortality events on the puaiohi population, in the absence of any other changes, we decreased previously estimated mortality at all life stages (VanderWerf et al. $2014)$ by $18 \%$, to a level at which they would provide a stable population projection (Fantle-Lepczyk et al. 2018).

In our predator-controlled release condition models, we assumed that predators would be controlled or removed from the release site. While puaiohi are potentially preyed upon by several species, rats are their major predator (Snetsinger et al. 2005), and mortality due to nest predation by rats is presumed to be the leading driver behind higher mortality rates seen in female puaiohi (VanderWerf et al. 2014). To reflect this difference in mortality, we decreased our estimates of female mortality by $1 / 4$, from 54 to $41 \%$ $(-13 \%)$. For the O'ahu 'elepaio, rat control has been shown to increase female survival by 10 to $27 \%$, which is in line with our estimates (VanderWerf 2009, VanderWerf et al. 2011). However, male puaiohi have an estimated annual survival rate of $71 \%$ (VanderWerf et al. 2014), so if predation on the nest is truly the major differential between male and female survival, removal of nest predators may in fact decrease female mortal- 
ity substantially more than we have estimated. Snetsinger et al. (2005) found that $50 \%$ more nests fledged young when rats were controlled. To allow for any overestimates in their experiment and other causes of nest failure, we increased the baseline number of successful attempts per year by $25 \%$. Experimental data quantifying the effects of rat predation on juvenile mortality do not exist. However, we presumed that removing rats would have less influence on juvenile mortality than on adult female mortality, as juveniles likely encounter a greater range of mortality effects, with the first days after fledging being the period they are most vulnerable. Thus, we decreased juvenile mortality by $10 \%$. We presumed that rat removal would not affect male mortality as much, since males do not incubate eggs (presumably, when females are most vulnerable to rat predation). Furthermore, removing rats should not impact the number of young fledged per attempt because a rat encountering a nest would likely consume both chicks, and thereby be classified as a failed attempt.

Our final release scenario looked at potential impacts of habitat improvements on the puaiohi. Under this scenario, we assumed that the release site would be managed such that invasive species would be controlled. This included rats which, while they act as predators, may also displace native food sources and potentially decrease food availability, particularly during the breeding season. Furthermore, we presumed that abundant native, species-appropriate food sources would exist. Snetsinger et al. (2005) saw an increase of $37.5 \%$ in annual nest attempts in wet years over drier years. Presumably this increase in nesting attempts was due in part to increased food supply, as well as decreased rat predation due to availability of alternative food sources. Snetsinger et al. (2005) further noted a $54.5 \%$ increase in the number fledged per attempt in the wet year compared to the mean number fledged over the entire $3 \mathrm{yr}$ of study. However, one very dry year reduces the mean number fledged, likely resulting in over estimations of this impact (Fantle-Lepczyk et al. 2016). Considering these points, we conservatively increased the number of successful nesting attempts per year and number of young fledged per attempt by 37.5 and $10 \%$, respectively, to reflect the greater food supply and decreased predation. Finally, we assumed that increased food supply and decreased predation could slightly decrease juvenile, male, and female mortality, so those parameters were accordingly adjusted by $10 \%$.

\subsection{Defining scenario outcomes}

We made an a priori decision that a newly established translocation scenario would be considered minimally successful if the new population attained at least 250 birds by $25 \mathrm{yr}$, had less than a $5 \%$ probability of extinction, and a growth rate greater than 0 . A sustainably successful scenario would create a new population of 500 or more birds with a $0 \%$ probability of extinction, and growth rate greater than 0.10 , a level of potential population increase that should allow some buffering for bad years. We tabulated how many scenarios achieved either level of success for each of the 3 output parameters of interest (growth rate, probability of extinction, and final population size). For the original source population, we also tabulated how many harvests of birds to be translocated failed over all 1000 iterations of each scenario, due to the unavailability of juvenile birds tomove. We defined failed harvest attempts over $10 \%$ as an unacceptable rate of failure, because at that point too few birds would be available to translocate to maximize the chances of creating a new population.

\subsection{Statistical analysis}

We tested the impact of harvest (for translocation to new population) on the original population via equivalency testing in Minitab (version 17.3.1). Equivalence testing is a statistical approach used to test if observations from 2 groups are similar enough to be biologically analogous. In equivalence testing, the null hypothesis is that the difference between the means is greater than a researcher-defined amount, which is referred to as 'interval of tolerable difference.' We set the limits at which we considered differences in baseline and model output differences from the baseline to be trivial as \pm 0.02 stochastic $r$ (essentially $\pm 2 \%$ annual growth rate), \pm 0.05 probability of extinction, and \pm 10 individuals or $\pm 10 \%$ of baseline individuals (whichever was greater) remaining at 25 yr. Under the scenarios with a declining original population, we also considered time to extinction, as most iterations within these went extinct, with \pm 3 yr considered trivial.

All statistical analyses aside from equivalency tests were performed in Systat 13. We used ANOVA with Bonferroni pairwise comparisons to evaluate the effects of initial harvest/population, number harvested/supplemented per event, harvest/supplementation interval, length of harvest/supplementation, 
Table 2. Minimally and sustainably successful regimes for establishing a translocated population of puaiohi

\begin{tabular}{|lccr|}
\hline Output parameter & $\begin{array}{c}\text { No. minimally } \\
\text { successful }\end{array}$ & $\begin{array}{c}\text { No. sustainably } \\
\text { successful }\end{array}$ & \multicolumn{1}{c|}{$\begin{array}{c}\text { Total } \\
\text { successful }\end{array}$} \\
\hline Growth rate (stochastic $r)$ & $80(74.1 \%)$ & $26(24.1 \%)$ & $106(98.1 \%)$ \\
Probability of extinction & $33(30.6 \%)$ & $17(15.7 \%)$ & $50(42.3 \%)$ \\
Population size at 25 yr & $21(19.4 \%)$ & $11(10.2 \%)$ & $32(29.6 \%)$ \\
Meets all 3 criteria & $22(20.4 \%)$ & $10(9.3 \%)$ & $32(29.6 \%)$ \\
\hline
\end{tabular}

tween initial populations of 10 and 20 individuals, most models required 10 years of supplementation (9 of 10), 20 individuals supplemented per translocation event (8 of 10), and yearly supplementation ( 7 of 10 ). Of the top 6 models, the same 3 translocation protocols appear twice, under both habitat-improvement and predator-controlled release conditions. All sustainable models required and release conditions on the growth rate, probability of extinction, time to extinction, and population size at 25 yr of both the original and new population. We used linear regressions to evaluate how number of times supplemented and number of individuals supplemented affected growth rate, probability of extinction, time to extinction, and population size at $25 \mathrm{yr}$ of both populations. We considered a $\mathrm{p} \leq 0.05$ as significant.

\section{RESULTS}

\subsection{Translocated population}

Nearly all translocation scenarios resulted in a positive growth rate for the new population, though only approximately $40 \%$ met the minimal criteria for probability of extinction and $30 \%$ for population size at $25 \mathrm{yr}$ (Table 2). Only 9\% met the sustainable criteria for all 3 output measures. Amongst these models, the majority ( 7 of 10) was under improved habitat release conditions, and the remaining 3 under predator-controlled release conditions (Table 3). While sustainable models were evenly split be- translocating $\geq 110$ birds, which represented the most bird-intensive scenarios modeled.

Comparisons amongst different levels in the initial population size, number supplemented per event, supplementation interval, and length of supplementation for the new population indicated that all but one resulted in significant differences in growth rate, probability of extinction, and population size at 25 yr (Table 4). Growth rates depended on initial supplementation parameter value, except that supplementation of 5 or 10 individuals per event were equivalent and resulted in lower growth rates than supplementing 20 individuals. For all other parameters, as the number of individuals and length of supplementation increased or supplementation interval decreased, the growth rate increased. For probability of extinction, supplementation of 10 or 20 individuals per event did not differ significantly, though supplementing 5 individuals resulted in a significantly higher extinction probability. In addition, supplementing every year or every other year did not yield significant differences in probability of extinction, while supplementing only every 5 yr did produce a significantly higher probability of extinction.

Table 3. Optimally successful supplementation regimes for establishing a translocated population of puaiohi. IH: improved habitat; PC: predator-controlled

\begin{tabular}{|lccccccccc|}
\hline $\begin{array}{l}\text { Initial } \\
\text { pop. }\end{array}$ & $\begin{array}{c}\text { No. } \\
\text { supple- } \\
\text { mented }\end{array}$ & $\begin{array}{c}\text { Supplement } \\
\text { interval } \\
\text { (yr) }\end{array}$ & $\begin{array}{c}\text { No. years } \\
\text { supple- } \\
\text { ment }\end{array}$ & $\begin{array}{c}\text { Release } \\
\text { condi- } \\
\text { tions }\end{array}$ & $\begin{array}{c}\text { No. times } \\
\text { supple- } \\
\text { mented }\end{array}$ & $\begin{array}{c}\text { Total no. } \\
\text { supple- } \\
\text { mented }\end{array}$ & $\begin{array}{c}\text { Mean } \pm \text { SD } \\
\text { growth rate } \\
\text { (stochastic } r \text { ) }\end{array}$ & $\begin{array}{c}\text { Probability } \\
\text { of } \\
\text { extinction }\end{array}$ & $\begin{array}{c}\text { Mean } \pm \text { SD } \\
\text { no. at } \\
25 \text { yr }\end{array}$ \\
\hline 10 & 20 & 2 & 10 & IH & 5 & 110 & $0.17 \pm 0.36$ & 0 & $662 \pm 289$ \\
20 & 20 & 1 & 10 & IH & 10 & 220 & $0.14 \pm 0.23$ & 0 & $647 \pm 299$ \\
10 & 20 & 1 & 10 & IH & 10 & 210 & $0.17 \pm 0.28$ & 0 & $629 \pm 301$ \\
10 & 20 & 2 & 10 & PC & 5 & 110 & $0.16 \pm 0.36$ & 0 & $540 \pm 287$ \\
20 & 20 & 1 & 10 & PC & 10 & 220 & $0.13 \pm 0.22$ & 0 & $538 \pm 292$ \\
10 & 20 & 1 & 10 & PC & 10 & 210 & $0.15 \pm 0.27$ & 0 & $516 \pm 282$ \\
20 & 20 & 1 & 5 & IH & 5 & 120 & $0.12 \pm 0.23$ & 0.004 & $501 \pm 320$ \\
20 & 10 & 1 & 10 & IH & 10 & 120 & $0.12 \pm 0.20$ & 0.002 & $494 \pm 310$ \\
20 & 20 & 2 & 10 & IH & 5 & 120 & $0.12 \pm 0.23$ & 0.002 & $483 \pm 318$ \\
10 & 10 & 1 & 10 & IH & 10 & 110 & $0.14 \pm 0.23$ & 0.002 & $451 \pm 304$ \\
\hline
\end{tabular}


Table 4. Translocated puaiohi population supplementation regime. ANOVA results for initial population, number of individuals supplemented, supplement interval, number of years supplemented, and release conditions, and linear regression results for number of times supplemented and total number of individuals supplemented. Values below $F$-statistic are least square means \pm SE. Different superscripts denote significant differences between groups based on Bonferroni post-hoc pairwise comparisons from the ANOVA

\begin{tabular}{|c|c|c|c|}
\hline & Growth rate (stochastic $r$ ) & Probability of extinction & Population size \\
\hline Initial population & $F_{1,106}=5.31, p=0.023$ & $F_{1,106}=5.92, \mathrm{p}=0.017$ & $F_{1,106}=1.05, p=0.308$ \\
\hline 10 & $0.08 \pm 0.01^{\mathrm{a}}$ & $0.13 \pm 0.02^{\mathrm{a}}$ & $177.65 \pm 20.76^{\mathrm{a}}$ \\
\hline 20 & $0.06 \pm 0.01^{\mathrm{b}}$ & $0.08 \pm 0.02^{\mathrm{b}}$ & $207.71 \pm 20.76^{\mathrm{a}}$ \\
\hline No. supplemented & $F_{2,105}=14.24, \mathrm{p}<0.001$ & $F_{2,105}=11.31, \mathrm{p}<0.001$ & $F_{2,105}=12.76, \mathrm{p}<0.001$ \\
\hline 5 & $0.05 \pm 0.01^{\mathrm{a}}$ & $0.17 \pm 0.02^{\mathrm{a}}$ & $122.02 \pm 23.02^{\mathrm{a}}$ \\
\hline 10 & $0.07 \pm 0.01^{a}$ & $0.10 \pm 0.02^{b}$ & $173.04 \pm 23.02^{\mathrm{a}}$ \\
\hline 20 & $0.1 \pm 0.01^{\mathrm{b}}$ & $0.04 \pm 0.02^{\mathrm{b}}$ & $282.97 \pm 23.02^{\mathrm{b}}$ \\
\hline Supplement interval & $F_{2,105}=23.33, p<0.001$ & $F_{2,105}=27.56, \mathrm{p}<0.001$ & $F_{2,105}=16.71, \mathrm{p}<0.001$ \\
\hline 1 yr & $0.10 \pm 0.01^{\mathrm{a}}$ & $0.03 \pm 0.02^{\mathrm{a}}$ & $278.41 \pm 22.36^{\mathrm{a}}$ \\
\hline $2 \mathrm{yr}$ & $0.08 \pm 0.01^{b}$ & $0.07 \pm 0.02^{\mathrm{a}}$ & $203.11 \pm 22.36^{\mathrm{a}}$ \\
\hline 5 yr & $0.04 \pm 0.01^{\mathrm{c}}$ & $0.20 \pm 0.02^{\mathrm{b}}$ & $96.52 \pm 22.36^{b}$ \\
\hline No. years supplemented & $F_{1,106}=8.59, \mathrm{p}=0.004$ & $F_{1,106}=12.52, \mathrm{p}<0.001$ & $F_{1,106}=6.67, \mathrm{p}=0.011$ \\
\hline 5 & $0.06 \pm 0.01^{a}$ & $0.15 \pm 0.02^{\mathrm{a}}$ & $155.73 \pm 20.23^{\mathrm{a}}$ \\
\hline 10 & $0.08 \pm 0.01^{\mathrm{b}}$ & $0.07 \pm 0.02^{\mathrm{b}}$ & $229.63 \pm 20.23^{\mathrm{b}}$ \\
\hline No. times supplemented & $F_{1,106}=56.36, \mathrm{p}<0.001$ & $F_{1,106}=52.33, \mathrm{p}<0.001$ & $F_{1,106}=44.77, \mathrm{p}<0.001$ \\
\hline $\mathrm{r}^{2}$ & 0.35 & 0.33 & 0.29 \\
\hline Slope & 0.01 & -0.02 & 28.29 \\
\hline Total no. supplemented & $F_{1,106}=94.96, p<0.001$ & $F_{1,106}=57.78, \mathrm{p}<0.001$ & $F_{1,106}=123.30, \mathrm{p}<0.001$ \\
\hline $\mathrm{r}^{2}$ & 0.47 & 0.35 & 0.54 \\
\hline Slope & 0.0006 & -0.002 & 2.35 \\
\hline Release conditions & $F_{2,105}=24.31, \mathrm{p}<0.001$ & $F_{2,105}=7.41, \mathrm{p}<0.001$ & $F_{2,105}=25.61, \mathrm{p}<0.001$ \\
\hline Stable mortality & $0.04 \pm 0.01^{\mathrm{a}}$ & $0.16 \pm 0.02^{\mathrm{a}}$ & $84.1 \pm 21.05^{\mathrm{a}}$ \\
\hline Predator-controlled & $0.08 \pm 0.01^{b}$ & $0.08 \pm 0.02^{\mathrm{b}}$ & $196.97 \pm 21.05^{\mathrm{b}}$ \\
\hline Improved habitat & $0.10 \pm 0.01^{\mathrm{c}}$ & $0.07 \pm 0.02^{\mathrm{b}}$ & $296.97 \pm 21.05^{\mathrm{c}}$ \\
\hline
\end{tabular}

As initial population size and length of supplementation increased, the probability of extinction decreased, though initial population size had no significant impact on population size at 25 yr. However, a significant difference existed in the population size at $25 \mathrm{yr}$, based on supplementing for 5 versus $10 \mathrm{yr}$. Supplementation of 20 birds per supplementation event yielded a significantly higher final population size than supplementing 5 or 10 birds per event, as did supplementing every year or every other year versus every $5 \mathrm{yr}$.

Both number of times and total number of individuals supplemented were significantly related to growth rate, probability of extinction, and population after 25 yr (Table 4); as supplementations increased, growth rate and final population size increased and probability of extinction decreased. Amongst the 3 types of release conditions, habitat improvement yielded the highest growth rate and greatest population size after $25 \mathrm{yr}$ and lowest (though not significantly different from predator removal) probability of extinctions. In all cases, the stable mortality scenarios yielded the lowest growth rates and population sizes, and significantly higher probabilities of extinction.

\subsection{Source population}

\subsubsection{Juvenile harvest}

While several scenarios successfully established translocated populations, harvest of juveniles for translocation predictably impacts the original population. Harvest scenarios had varying consequences for the original donor population, with the most detrimental effects seen in the declining wild population. Though all scenarios were equivalent to the declining source baseline in terms of probability of extinction $(p>0.97)$ and population size at $25 \mathrm{yr}$ ( $\mathrm{n}<0.4$ individuals), this was likely due to the extremely high probability of extinction and low population sizes exhibited by all the declining scenarios. However, $89 \%$ of translocation models did not exhibit growth rates and $22 \%$ did not have a mean time to extinction equivalent to the declining baseline (Table 5a). All sustainably successful translocation scenarios (Table 3) exhibited significantly lower growth rates and shorter mean times to extinction in a harvested original population than in a non-harvested one. Furthermore, when harvesting 
Table 5. Equivalency tests for the original puaiohi population under (a) data-driven declining conditions and (b) stable conditions. Only models that exhibited non-equivalency in at least 1 output parameter are included. Models that were equivalent in all model output parameters are excluded from the tables

\begin{tabular}{|c|c|c|c|c|c|c|c|}
\hline $\begin{array}{l}\text { Initial } \\
\text { pop. }\end{array}$ & $\begin{array}{c}\text { No. } \\
\text { harvested }\end{array}$ & $\begin{array}{l}\text { Harvest } \\
\text { interval }\end{array}$ & $\begin{array}{l}\text { No. years } \\
\text { harvested }\end{array}$ & $\begin{array}{l}\text { Mean } \pm \mathrm{SD} \\
\text { growth rate } \\
(\text { stochastic } r \text { ) }\end{array}$ & $\mathrm{p}$-value & $\begin{array}{l}\text { Mean } \pm \mathrm{SD} \\
\text { time to } \\
\text { extinction }\end{array}$ & $\mathrm{p}$-value \\
\hline (a) Baseline-declini & hing & & & $-0.267 \pm 0.179$ & & $16.7 \pm 3.5$ & \\
\hline 10 & 5 & $1 \mathrm{yr}$ & 5 & $-0.279 \pm 0.208$ & 0.194 & $15.8 \pm 3.6$ & $<0.001$ \\
\hline 10 & 5 & $1 \mathrm{yr}$ & 10 & $-0.304 \pm 0.207$ & 0.978 & $13.2 \pm 3.7$ & 0.999 \\
\hline 10 & 5 & $2 \mathrm{yr}$ & 10 & $-0.284 \pm 0.209$ & 0.387 & $15.2 \pm 3.8$ & $<0.001$ \\
\hline 10 & 5 & $5 \mathrm{yr}$ & 10 & $-0.278 \pm 0.214$ & 0.157 & $15.7 \pm 3.6$ & $<0.001$ \\
\hline 10 & 10 & $1 \mathrm{yr}$ & 5 & $-0.286 \pm 0.211$ & 0.441 & $15.5 \pm 3.8$ & $<0.001$ \\
\hline 10 & 10 & $1 \mathrm{yr}$ & 10 & $-0.328 \pm 0.216$ & 1.000 & $11.9 \pm 3.2$ & 1.000 \\
\hline 10 & 10 & $2 \mathrm{yr}$ & 5 & $-0.276 \pm 0.208$ & 0.105 & $16.1 \pm 3.6$ & $<0.001$ \\
\hline 10 & 10 & $2 \mathrm{yr}$ & 10 & $-0.294 \pm 0.216$ & 0.785 & $14.5 \pm 3.7$ & $<0.001$ \\
\hline 10 & 10 & $5 \mathrm{yr}$ & 5 & $-0.274 \pm 0.212$ & 0.063 & $16.2 \pm 3.7$ & $<0.001$ \\
\hline 10 & 10 & $5 \mathrm{yr}$ & 10 & $-0.285 \pm 0.223$ & 0.425 & $15.3 \pm 3.5$ & $<0.001$ \\
\hline 10 & 20 & $1 \mathrm{yr}$ & 5 & $-0.308 \pm 0.226$ & 0.990 & $14.4 \pm 3.9$ & $<0.001$ \\
\hline 10 & 20 & $1 \mathrm{yr}$ & 10 & $-0.354 \pm 0.211$ & 1.000 & $10.6 \pm 2.6$ & 1.000 \\
\hline 10 & 20 & $2 \mathrm{yr}$ & 5 & $-0.290 \pm 0.211$ & 0.617 & $15.3 \pm 3.8$ & $<0.001$ \\
\hline 10 & 20 & $2 \mathrm{yr}$ & 10 & $-0.322 \pm 0.223$ & 1.000 & $13.2 \pm 3.5$ & 0.999 \\
\hline 10 & 20 & $5 \mathrm{yr}$ & 5 & $-0.278 \pm 0.211$ & 0.155 & $16.1 \pm 3.6$ & $<0.001$ \\
\hline 10 & 20 & $5 \mathrm{yr}$ & 10 & $-0.291 \pm 0.223$ & 0.687 & $15.1 \pm 3.5$ & $<0.001$ \\
\hline 20 & 5 & $1 \mathrm{yr}$ & 5 & $-0.275 \pm 0.208$ & 0.089 & $16 \pm 3.6$ & $<0.001$ \\
\hline 20 & 5 & $1 \mathrm{yr}$ & 10 & $-0.308 \pm 0.211$ & 0.991 & $13.2 \pm 3.5$ & 0.999 \\
\hline 20 & 5 & $2 \mathrm{yr}$ & 5 & $-0.276 \pm 0.208$ & 0.094 & $16.2 \pm 3.6$ & $<0.001$ \\
\hline 20 & 5 & $2 \mathrm{yr}$ & 10 & $-0.285 \pm 0.210$ & 0.392 & $15.4 \pm 3.8$ & $<0.001$ \\
\hline 20 & 5 & $5 \mathrm{yr}$ & 10 & $-0.280 \pm 0.215$ & 0.446 & $15.5 \pm 3.6$ & $<0.001$ \\
\hline 20 & 10 & $1 \mathrm{yr}$ & 5 & $-0.286 \pm 0.212$ & 0.224 & $15.4 \pm 3.8$ & $<0.001$ \\
\hline 20 & 10 & $1 \mathrm{yr}$ & 10 & $-0.331 \pm 0.216$ & 1.000 & $11.8 \pm 3.0$ & 1.000 \\
\hline 20 & 10 & $2 \mathrm{yr}$ & 5 & $-0.276 \pm 0.211$ & 0.113 & $16 \pm 3.7$ & $<0.001$ \\
\hline 20 & 10 & $2 \mathrm{yr}$ & 10 & $-0.300 \pm 0.215$ & 0.928 & $14.3 \pm 3.6$ & $<0.001$ \\
\hline 20 & 10 & $5 \mathrm{yr}$ & 10 & $-0.281 \pm 0.218$ & 0.254 & $15.7 \pm 3.6$ & $<0.001$ \\
\hline 20 & 20 & $1 \mathrm{yr}$ & 5 & $-0.311 \pm 0.224$ & 0.995 & $14.2 \pm 3.8$ & 0.001 \\
\hline 20 & 20 & $1 \mathrm{yr}$ & 10 & $-0.357 \pm 0.203$ & 1.000 & $10.1 \pm 2.3$ & 1.000 \\
\hline 20 & 20 & $2 \mathrm{yr}$ & 5 & $-0.290 \pm 0.214$ & 0.654 & $15.2 \pm 3.7$ & $<0.001$ \\
\hline 20 & 20 & $2 \mathrm{yr}$ & 10 & $-0.325 \pm 0.221$ & 1.000 & $13.2 \pm 3.3$ & 0.999 \\
\hline 20 & 20 & $5 \mathrm{yr}$ & 5 & $-0.275 \pm 0.211$ & 0.087 & $16 \pm 3.7$ & $<0.001$ \\
\hline 20 & 20 & $5 \mathrm{yr}$ & 10 & $-0.290 \pm 0.224$ & 0.638 & $15.1 \pm 3.4$ & $<0.001$ \\
\hline (b) Baseline-stable & & & & $-0.002 \pm 0.129$ & & $492 \pm 256$ & \\
\hline 10 & 10 & $1 \mathrm{yr}$ & 5 & $-0.008 \pm 0.131$ & 0.007 & $453 \pm 262$ & 0.173 \\
\hline 10 & 10 & $1 \mathrm{yr}$ & 10 & $-0.013 \pm 0.133$ & 0.061 & $421 \pm 260$ & 0.964 \\
\hline 10 & 20 & $1 \mathrm{yr}$ & 5 & $-0.010 \pm 0.131$ & 0.018 & $438 \pm 256$ & 0.653 \\
\hline 10 & 20 & $1 \mathrm{yr}$ & 10 & $-0.031 \pm 0.145$ & 0.943 & $329 \pm 259$ & 1.000 \\
\hline 10 & 20 & $2 \mathrm{yr}$ & 10 & $-0.012 \pm 0.134$ & 0.048 & $432 \pm 269$ & 0.819 \\
\hline 20 & 5 & $1 \mathrm{yr}$ & 10 & $-0.008 \pm 0.132$ & 0.002 & $448 \pm 270$ & 0.320 \\
\hline 20 & 10 & $1 \mathrm{yr}$ & 5 & $-0.007 \pm 0.131$ & 0.006 & $449 \pm 263$ & 0.285 \\
\hline 20 & 10 & $1 \mathrm{yr}$ & 10 & $-0.013 \pm 0.134$ & 0.080 & $410 \pm 266$ & 0.997 \\
\hline 20 & 10 & $2 \mathrm{yr}$ & 5 & $-0.005 \pm 0.132$ & 0.002 & $461 \pm 262$ & 0.051 \\
\hline 20 & 20 & $1 \mathrm{yr}$ & 5 & $-0.011 \pm 0.132$ & 0.035 & $430 \pm 269$ & 0.856 \\
\hline 20 & 20 & $1 \mathrm{yr}$ & 10 & $-0.032 \pm 0.148$ & 0.950 & $328 \pm 260$ & 1.000 \\
\hline 20 & 20 & $2 \mathrm{yr}$ & 5 & $-0.007 \pm 0.130$ & 0.006 & $450 \pm 267$ & 0.247 \\
\hline 20 & 20 & $2 \mathrm{yr}$ & 10 & $-0.011 \pm 0.132$ & 0.028 & $433 \pm 266$ & 0.799 \\
\hline
\end{tabular}

for translocation from a declining baseline there were model runs in which too few juveniles existed to harvest (Table 6). Specifically, in $44 \%$ of our translocation scenarios, $10 \%$ or more of all translocations attempted across all scenario iterations resulted in an inability to harvest sufficient juveniles for translocation, which exceeds the level above which we deemed unacceptable. All but 2 of the models in which harvest lasted 10 years exhibited failure rates over $10 \%$. 
Table 6. Total number of puaiohi harvests attempted and failed per 1000 scenario iterations under declining baseline conditions

\begin{tabular}{|lcccrrr|}
\hline $\begin{array}{l}\text { Initial } \\
\text { harvest }\end{array}$ & $\begin{array}{c}\text { No. } \\
\text { harvested }\end{array}$ & $\begin{array}{c}\text { Harvest } \\
\text { interval }\end{array}$ & $\begin{array}{c}\text { No. years } \\
\text { harvested }\end{array}$ & $\begin{array}{c}\text { Total } \\
\text { failed }\end{array}$ & $\begin{array}{c}\text { Total } \\
\text { attempted }\end{array}$ & $\begin{array}{c}\% \\
\text { failed }\end{array}$ \\
\hline 20 & 20 & $1 \mathrm{yr}$ & 10 & 86237 & 200000 & 43.12 \\
10 & 20 & $1 \mathrm{yr}$ & 10 & 84094 & 200000 & 42.05 \\
20 & 20 & $5 \mathrm{yr}$ & 10 & 15832 & 40000 & 39.58 \\
10 & 20 & $5 \mathrm{yr}$ & 10 & 15363 & 40000 & 38.41 \\
20 & 20 & $2 \mathrm{yr}$ & 10 & 27977 & 100000 & 27.98 \\
10 & 20 & $2 \mathrm{yr}$ & 10 & 27458 & 100000 & 27.46 \\
20 & 10 & $1 \mathrm{yr}$ & 10 & 25942 & 100000 & 25.94 \\
10 & 10 & $1 \mathrm{yr}$ & 10 & 25115 & 100000 & 25.12 \\
10 & 10 & $5 \mathrm{yr}$ & 10 & 4959 & 20000 & 24.80 \\
20 & 10 & $5 \mathrm{yr}$ & 10 & 4788 & 20000 & 23.94 \\
10 & 5 & $5 \mathrm{yr}$ & 10 & 1634 & 10000 & 16.34 \\
20 & 5 & $5 \mathrm{yr}$ & 10 & 1625 & 10000 & 16.25 \\
20 & 5 & $1 \mathrm{yr}$ & 10 & 7422 & 50000 & 14.84 \\
20 & 10 & $2 \mathrm{yr}$ & 10 & 7397 & 50000 & 14.79 \\
10 & 5 & $1 \mathrm{yr}$ & 10 & 7388 & 50000 & 14.78 \\
10 & 10 & $2 \mathrm{yr}$ & 10 & 6673 & 50000 & 13.35 \\
\hline
\end{tabular}

nal declining baseline population regardless of translocation scenario. In the case of the number of individuals harvested per harvesting event, however, there were notable differences. While the number of individuals harvested per event did not significantly impact the probability of, or time to extinction, it did affect the growth rate and population size at $25 \mathrm{yr}$ in the original declining baseline population. Specifically, harvesting 20 individuals resulted in a significantly lower growth rate than did harvesting 5 individuals, and a significantly smaller population size at $25 \mathrm{yr}$ than did harvesting 5 or 10 individuals. Harvest interval also had effects on the original declining baseline population. That is, harvesting every year resulted in a

Initial harvest, number of individuals harvested per event, harvest interval, and number of years harvested varied in how they affected the declining baseline population (Table 7). For initial harvest, we found no effect on the original declining baseline population across translocation scenarios. Specifically, growth rates, probabilities of extinction, population sizes at $25 \mathrm{yr}$, and times to extinction were similar to the origi- significantly lower growth rate and shorter time to extinction than harvesting every other year or every $5 \mathrm{yr}$. Harvesting every year also produced a significantly higher probability of extinction and lower population size at 25 yr than harvesting every 5 yr. Finally, the number of years harvested had differential effects on the original declining baseline population. Harvesting for $10 \mathrm{yr}$ led to a significantly lower growth rate,

Table 7. Declining puaiohi source population harvest regime. ANOVA results for initial harvest, number of individuals harvested, harvest interval, and number of years harvested, and linear regression results for number of times harvested and total number of individuals harvested. Values below F-statistic are least square means \pm SE. Different superscripts denote significant differences between groups based on Bonferroni post-hoc pairwise comparisons from the ANOVA

\begin{tabular}{|lcccc|}
\hline & Growth rate (stochastic $r$ ) & Probability of extinction & Population size & Time to extinction \\
\hline Initial harvest & $F_{1,34}=0.01, \mathrm{p}=0.924$ & $F_{1,34}=0.11, \mathrm{p}=0.746$ & $F_{1,34}=0.02, \mathrm{p}=0.896$ & $F_{1,34}=0.01, \mathrm{p}=0.937$ \\
10 & $-0.29 \pm 0.01^{\mathrm{a}}$ & $0.98 \pm 0.002^{\mathrm{a}}$ & $0.20 \pm 0.02^{\mathrm{a}}$ & $14.82 \pm 0.39^{\mathrm{a}}$ \\
20 & $-0.29 \pm 0.01^{\mathrm{a}}$ & $0.98 \pm 0.002^{\mathrm{a}}$ & $0.19 \pm 0.02^{\mathrm{a}}$ & $14.78 \pm 0.39^{\mathrm{a}}$ \\
No. harvested & $F_{2,33}=4.88, \mathrm{p}=0.014$ & $F_{2,33}=2.16, \mathrm{p}=0.131$ & $F_{2,33}=3.39, \mathrm{p}=0.046$ & $F_{2,33}=2.39, \mathrm{p}=0.107$ \\
5 & $-0.28 \pm 0.01^{\mathrm{a}}$ & $0.98 \pm 0.003^{\mathrm{a}}$ & $0.23 \pm 0.03^{\mathrm{a}}$ & $15.44 \pm 0.46^{\mathrm{a}}$ \\
10 & $-0.29 \pm 0.01^{\mathrm{ab}}$ & $0.98 \pm 0.003^{\mathrm{a}}$ & $0.23 \pm 0.03^{\mathrm{a}}$ & $14.92 \pm 0.46^{\mathrm{a}}$ \\
20 & $-0.31 \pm 0.01^{\mathrm{b}}$ & $0.99 \pm 0.003^{\mathrm{a}}$ & $0.14 \pm 0.03^{\mathrm{b}}$ & $14.04 \pm 0.46^{\mathrm{a}}$ \\
Harvest interval & $F_{2,33}=8.53, \mathrm{p}=0.001$ & $F_{2,33}=7.68, \mathrm{p}=0.002$ & $F_{2,33}=5.69, \mathrm{p}=0.007$ & $F_{2,33}=9.00, \mathrm{p}=0.001$ \\
1 yr & $-0.31 \pm 0.01^{\mathrm{a}}$ & $0.99 \pm 0.002^{\mathrm{a}}$ & $0.13 \pm 0.03^{\mathrm{a}}$ & $13.51 \pm 0.39^{\mathrm{a}}$ \\
2 yr & $-0.29 \pm 0.01^{\mathrm{b}}$ & $0.98 \pm 0.002^{\mathrm{ab}}$ & $0.21 \pm 0.03^{\mathrm{ab}}$ & $15.07 \pm 0.39^{\mathrm{b}}$ \\
5 yr & $-0.28 \pm 0.01^{\mathrm{b}}$ & $0.98 \pm 0.002^{\mathrm{b}}$ & $0.25 \pm 0.03^{\mathrm{b}}$ & $15.83 \pm 0.39^{\mathrm{b}}$ \\
No. years harvested & $F_{1,34}=8.59, \mathrm{p}=0.001$ & $F_{1,34}=22.00, \mathrm{p}<0.001$ & $F_{1,34}=30.59, \mathrm{p}<0.001$ & $F_{1,34}=18.69, \mathrm{p}<0.001$ \\
5 & $-0.28 \pm 0.004^{\mathrm{a}}$ & $0.98 \pm 0.002^{\mathrm{a}}$ & $0.26 \pm 0.02^{\mathrm{a}}$ & $15.77 \pm 0.32^{\mathrm{a}}$ \\
10 & $-0.31 \pm 0.004^{\mathrm{b}}$ & $0.99 \pm 0.002^{\mathrm{b}}$ & $0.13 \pm 0.02^{\mathrm{b}}$ & $13.83 \pm 0.32^{\mathrm{b}}$ \\
No. times harvested & $F_{1,34}=63.77, \mathrm{p}<0.001$ & $F_{1,34}=49.04, \mathrm{p}<0.001$ & $F_{1,34}=51.54, \mathrm{p}<0.001$ & $F_{1,34}=157.17, \mathrm{p}<0.001$ \\
$\mathrm{r}^{2}$ & 0.65 & 0.59 & 0.6 & 0.82 \\
Slope & -0.01 & 0.0001 & -0.03 & -0.03 \\
Total no. harvested & $F_{1,34}=323.30, \mathrm{p}<0.001$ & $F_{1,34}=54.08, \mathrm{p}<0.001$ & $F_{1,34}=57.70, \mathrm{p}<0.001$ & $F_{1,34}=101.92, \mathrm{p}<0.001$ \\
r $^{2}$ & 0.9 & 0.61 & 0.63 & 0.75 \\
Slope & -0.0004 & 0.002 & -0.002 & -0.48
\end{tabular}


higher probability of extinction, smaller population size after $25 \mathrm{yr}$, and shorter time to extinction than did harvesting for $5 \mathrm{yr}$. As both total number of times harvested and total number of individuals harvested increased, growth rate, population size after $25 \mathrm{yr}$ and time to extinction decreased, and probability of extinction increased.

In the case of harvesting juveniles for translocation from our stable source population, we were unable to test for equivalency to baseline in terms of probability of extinction and mean time to extinction, due to lack of scenarios and iterations within scenarios that went extinct. However, translocation scenarios did differ from the stable source population in terms of the donor population size at $25 \mathrm{yr}$ for $36 \%$ of scenarios and growth rate in $11 \%$ of scenarios (Table 5b). Again, all sustainably successful translocation scenarios (Table 3) were amongst the non-equivalent models.

As with findings for the declining baseline population, the initial harvest, the number of individuals harvested per event, the harvest interval, and number of years harvested varied in how they affected the stable baseline population (Table 8). Initial harvest had no significant impact on probability of extinction, growth rate, or population size at $25 \mathrm{yr}$. The number of individuals harvested per event did not relate to 06robability of extinction, but it did relate to growth rate and population size, such that harvesting 5 individuals per event was significantly different from harvesting 20. Harvest interval had no significant impact on probability of extinction, though harvesting every year or every other year produced a significantly lower growth rate than did harvesting every fifth year. Harvesting every year also produced a significantly lower population size at $25 \mathrm{yr}$ than did harvesting every other or every fifth year. Furthermore, the number of years harvested did not significantly affect growth rate, probability of extinction, or population size at $25 \mathrm{yr}$ in the stable population. As number of times harvested or total number of juveniles harvested increased, growth rate declined, probability of extinction increased, and population size decreased.

\subsubsection{Rear and release}

In contrast to harvesting juveniles, harvesting eggs to rear and release had considerably less impact on the source population. In fact, all models for the declining baseline scenarios were equivalent for all 4 metrics (growth rate, probability of extinction, population size at $25 \mathrm{yr}$ and mean time to extinction). For the stable baseline models, all were equivalent in terms of growth rate and population size; we were unable to test probability of and time to extinction, since most scenarios and iterations within scenarios

Table 8. Stable puaiohi source population harvest regime. ANOVA results for initial harvest, number of individuals harvested, harvest interval, and number of years harvested, and linear regression results for number of times harvested and total number of individuals harvested. Values below F-statistic are least square means \pm SE. Different superscripts denote significant differences between groups based on Bonferroni post-hoc pairwise comparisons from the ANOVA

\begin{tabular}{|lccc|}
\hline & Growth rate (stochastic $r$ ) & Probability of extinction & Population size at 25 yr \\
\hline Initial harvest & $F_{1,34}=0.01, \mathrm{p}=0.906$ & $F_{1,34}=0.02, \mathrm{p}=0.877$ & $F_{1,34}=0.28, \mathrm{p}=0.602$ \\
10 & $-0.01 \pm 0.001^{\mathrm{a}}$ & $0.002 \pm 0.001^{\mathrm{a}}$ & $460.16 \pm 9.07^{\mathrm{a}}$ \\
20 & $-0.01 \pm 0.001^{\mathrm{a}}$ & $0.002 \pm 0.001^{\mathrm{a}}$ & $453.41 \pm 9.07^{\mathrm{a}}$ \\
No. harvested & $F_{2,33}=5.36, \mathrm{p}=0.009$ & $F_{2,33}=2.22, \mathrm{p}=0.125$ & $F_{2,33}=5.59, \mathrm{p}=0.008$ \\
5 & $-0.003 \pm 0.002^{\mathrm{a}}$ & $0.001 \pm 0.001^{\mathrm{a}}$ & $476.08 \pm 9.79^{\mathrm{a}}$ \\
10 & $-0.006 \pm 0.002^{\mathrm{ab}}$ & $0.001 \pm 0.001^{\mathrm{a}}$ & $463.15 \pm 9.79^{\mathrm{ab}}$ \\
20 & $-0.011 \pm 0.002^{\mathrm{b}}$ & $0.004 \pm 0.001^{\mathrm{a}}$ & $431.13 \pm 9.79^{\mathrm{b}}$ \\
Harvest interval & $F_{2,33}=8.17, \mathrm{p}=0.001$ & $F_{2,33}=2.57, \mathrm{p}=0.092$ & $F_{2,33}=9.54, \mathrm{p}<0.001$ \\
1 yr & $-0.01 \pm 0.002^{\mathrm{a}}$ & $0.004 \pm 0.001^{\mathrm{a}}$ & $425.98 \pm 9.02^{\mathrm{a}}$ \\
2 yr & $-0.006 \pm 0.002^{\mathrm{a}}$ & $0.001 \pm 0.001^{\mathrm{a}}$ & $464.21 \pm 9.02^{\mathrm{b}}$ \\
5 yr & $-0.003 \pm 0.002^{\mathrm{b}}$ & $F_{1,34}=3.76, \mathrm{p}=0.061$ & $480.17 \pm 9.02^{\mathrm{b}}$ \\
No. years harvested & $F_{1,34}=3.97, \mathrm{p}=0.054$ & $0.0002 \pm 0.001^{\mathrm{a}}$ & $F_{1,34}=3.88, \mathrm{p}=0.057$ \\
5 & $-0.005 \pm 0.002^{\mathrm{a}}$ & $0.003 \pm 0.001^{\mathrm{a}}$ & $468.81 \pm 8.63^{\mathrm{a}}$ \\
10 & $-0.01 \pm 0.002^{\mathrm{a}}$ & $F_{1,34}=13.72, \mathrm{p}<0.001$ & $444.77 \pm 8.63^{\mathrm{a}}$ \\
No. times harvested & $F_{1,34}=34.49, \mathrm{p}<0.001$ & 0.29 & $F_{1,34}=38.70, \mathrm{p}<0.001$ \\
$\mathrm{r}^{2}$ & 0.50 & 0.001 & 0.53 \\
Slope & -0.002 & $F_{1,34}=58.05, \mathrm{p}<0.001$ & -9.37 \\
Total no. harvested & $F_{1,34}=451.90, \mathrm{p}<0.001$ & 0.63 & $F_{1,34}=503.14, \mathrm{p}<0.001$ \\
r $^{2}$ & 0.93 & 0.0001 & 0.94 \\
Slope & -0.0001 & & -0.77 \\
\end{tabular}


did not go extinct. Furthermore, no element of our translocation regimes (initial population, number harvested per event, harvest interval, length of harvest, total number of times harvested, or total number supplemented) had any significant relationship with growth rate, probability of extinction, population size at $25 \mathrm{yr}$, or mean time to extinction.

\section{DISCUSSION}

Of the 108 possible models for our translocated population, only 32 met our overall criteria for success, and only 10 were sustainably successful. Amongst these 10, 7 occurred under habitat improvement release conditions and 3 under predatorcontrolled release conditions. Notably, none of our sustainably successful models occurred under stable mortality release conditions.

In general, initial population size did not have a major effect on translocation outcome, but as more individuals were supplemented per event, supplemented more frequently, or supplemented over 10 rather than $5 \mathrm{yr}$, establishing a new population was more likely to be successful. In terms of release conditions, better results were generally achieved under habitat improvement conditions, which included both control of predators and restored habitat, though positive results were also achieved under just predator-controlled conditions. Since habitat improvement in this scenario includes both control of predators and restored habitat, it understandably results in a more favorable outcome. The stable mortality models were uniformly less successful than the other 2 release conditions.

Unfortunately, when juveniles are harvested for translocation, the models providing sustainable translocation success for a new population had the greatest impacts on the source population. Regardless of whether we modeled the existing source population as a declining population or as a stable population, the 7 sustainably successful translocation regimes were all among the models that were not equivalent to baseline conditions ( $\mathrm{n}=32,13$, respectively, Table 5). These 7 models negatively impacted the source population such that growth rates, population size at $25 \mathrm{yr}$, or mean time to extinction was no longer comparable to a population that sustained no harvest for translocation. Furthermore, as supplementation levels increased and occurred over a longer time frame, an increasing percentage of translocations failed due to lack of juveniles to harvest in the datadriven declining baseline models.
Given that the more successful a translocation regime was at establishing a new population, the more detrimental it was to the original population if juveniles were harvested, it may not be possible to build a new population of puaiohi from juveniles within the existing wild flock. Although the wild flock has been presumed stable in recent years (USFWS 2006, Crampton et al. 2017), recent models based on best available data indicate that it may in fact be declining (Fantle-Lepczyk et al. 2018). Given the profound difference in effects of harvest based on source population, understanding more accurately the actual dynamics of the wild population is of great importance prior to any translocation project that involves regular removal of juveniles.

In contrast to harvest of juveniles, removal of eggs with the intent of rearing and releasing at the juvenile stage appears to have negligible effect on the source population, regardless of its current dynamics. This method offers an opportunity that would decrease the impact on the wild population and allow for higher rates of supplementation. If continued harvest of eggs or individuals from the wild population is deemed undesirable, the only other option would be to re-initiate a captive breeding program for the puaiohi. Under the original captive breeding program, the entire captive population was derived from 15 eggs (of 19 collected), and produced an average of almost 16 releasable birds per year (Switzer et al. 2013). As this number of releasable birds is comparable to many of our sustainably successful models, captive breeding may offer a reliable and sufficient source of birds to ensure translocation success. Previous reintroductions were perceived to have met with limited success due to lack of genetic diversity, understanding of puaiohi life history and demographics, and ecosystem restoration (Switzer et al. 2013). A more robust captive breeding and reintroduction program could be achieved by incorporating more genetic diversity into the founder flock, investing in research into puaiohi life history and demographics, and increased effort to restore habitat.

Though our results have implications for puaiohi conservation, several caveats must be recognized. First, PVAs are often criticized for their dependence on the large amounts of quality data necessary to provide accurate output (Hamilton \& Moller 1995, Taylor 1995, Groom \& Pascual 1998, Ludwig 1996, Ellner et al. 2002). Although we have used the best available data to populate our models, this study faces the same limitations. However, the utility of PVA is less in the actual numbers it provides, but rather as a means of comparing options through con- 
sidering relative extinction risks, either between subpopulations of a species or between alternative management actions (Lindenmayer \& Possingham 1996, Akçakaya \& Sjögren-Gulve 2000, Brook et al. 2000, Morris \& Doak 2002).

A second caveat is that, though we have tried to provide realistic estimates of puaiohi demographics under a suite of release conditions, these estimates are not based upon real locations. Success of a translocation program is dependent upon identifying available locations that approximate the conditions modeled in our improved habitat or predator-controlled release conditions. In order to find a suitable release site, extensive survey work and research will be required, and significant habitat improvement may be necessary. Should puaiohi be translocated, one of the higher elevation Hawaiian Islands, such as Maui or Hawai'i, will likely be considered (Hawai'i Forest Bird Conservation Forum, September 24-25, 2015). Although agency consultation would utilize a structured decision framework to select an appropriate location, Maui is the likely candidate as its native thrush, the 'āmaui Myadestes woahensis is extinct, while the congeneric ōma'o $M$. obscurus is still extant on the island of Hawai'i.

There are many factors that will need to be considered before puaiohi can be translocated, including sufficient nesting sites, food availability, and protection from predators, invasive competitors, and disease. On Kaua'i, puaiohi live in forested ravines along streams (Snetsinger et al. 1999, USFWS 2006) in wet native montane 'ōhi'a Metrosideros polymorpha forest, with subdominant 'ōhia ha Syzygium sandwicensis and 'ōlapa Cheirodendron spp., and a rich understory of native plants including 'ōhelo Vaccinium calycinum and kanawao Broussaisia arguta. One concern is that these stream cuts may not occur with sufficient frequency on other islands to support an adequate population. However, puaiohi may not be obligate stream nesters, as they will nest in trees and historically, they were more widespread and may have occurred down to sea level where steam cuts are less common or absent (Perkins 1903, James \& Olson 1991, Burney et al. 2001). Reintroduction to predator-free or -controlled areas may allow puaiohi to utilize a greater diversity of nesting sites. In addition to meeting conditions for nesting sites, another habitat requirement that will need to be considered is food availability. Fruit eaten include 'ōlapa species, 'ōhia ha, kanawao, 'ōhelo, pa'iniu Astelia spp., pūkiawe Styphelia tameiameiae, kāwa'u Ilex anomala, and pilo Coprosma spp. (Snetsinger et al. 1999). While puaiohi are primarily frugivorous, arthropods are also an important dietary component, especially during the breeding season (Richardson \& Bowles 1964, Kepler \& Kepler 1983, Scott et al. 1986, Snetsinger et al. 1999). Since predation by alien mammals (e.g. Rattus spp.) currently inhibits productivity, and competition for food with introduced insects, birds, and mammals may also have negative impacts (Snetsinger et al. 1999, 2005), it may prove necessary to control these invasive species in order to maximize translocation success. Finally, malarial infection is a concern, though the extent of its impact on the population remains unclear. Thus, puaiohi should be released into malaria refugia.

Our results suggest that tradeoffs exist in establishing a second puaiohi population. If a juvenile harvest approach is used to successfully establish a second population, it is likely to negatively impact the existing wild population. Alternatively, the harvest of eggs to be reared and released seasonally and/or the re-establishment of a captive breeding program may offer a way to both preserve the wild population and allow for a sufficient supply of birds for supplementing a new population.

Determining if translocation is successful will require long-term monitoring of the newly established population. Specifically, it would be highly beneficial to follow a mark recapture approach to evaluate population size, growth rate, and occupancy. Ultimately, however, the success of the reintroduction program is dependent upon ecosystem restoration, both in the source and translocation habitats. As novel, and perhaps more drastic, management techniques are considered, active management and restoration of puaiohi habitat must also continue to occur to preserve this rare species for future generations.

Acknowledgements. Data used in this study were collected by the Kaua'i Forest Bird Recovery Project (KFBRP), a cooperative project of the Pacific Cooperative Studies Unit of the University of Hawai'i at Mānoa and the State of Hawai'i Department of Land and Natural Resources, Division of Forestry and Wildlife. Funding for KFBRP is provided by the US Fish and Wildlife Service and the State of Hawai'i. We thank the staff members and field technicians of KFBRP for their efforts to collect data for this project as well as 2 anonymous reviewers and J. Michael Reed for comments on the draft manuscript.

\section{LITERATURE CITED}

Akçakaya HR (2005) RAMAS Metapop: viability analysis for stage-structured metapopulations (version 5). Applied Biomathematics, Setauket, NY

Akçakaya HR, Sjögren-Gulve P (2000) Population viability analysis in conservation planning: an overview. Ecol Bull 48:9-21 
Akçakaya HR, Burgman MA, Ginzburg LR (1999) Applied population ecology, 2nd edn. Sinauer Associates, Sunderland, MA

*Beissinger SR, Westphal MI (1998) On the use of demographic models of population viability in endangered species management. J Wildl Manag 62:821-841

* Benning TL, LaPointe DA, Atkinson CT, Vitousek PM (2002) Interactions of climate change with biological invasions and land use in the Hawaiian Islands: Modeling the fate of endemic birds using a geographic information system. Proc Natl Acad Sci USA 99:14246-14249

Brook BW, O'Grady JJ, Chapman AP, Burgman MA, Akçakaya HR, Frankham R (2000) Predictive accuracy of population viability analysis in conservation biology. Nature 404:385-387

Burney DA, James HF, Burney LP, Olson SL and others (2001) Fossil evidence for a diverse biota from Kaua'i and its transformation since human arrival. Ecol Monogr 71: 615-641

Conant S, Morin M (2001) Why isn't the Nihoa millerbird extinct? Stud Avian Biol 22:338-346

Conant S, Pratt HD, Shallenberger RJ (1998) Reflections on a 1975 ornithological expedition to the lost world of the Alaka'i and other notes on the natural history, systematics and conservation of Kaua'i birds. Wilson Bull 110: $1-22$

* Crampton LH, Brinck KW, Pias KE, Heindl BAP, Savre T, Diegmann JS, Paxton EH (2017) Linking occupancy surveys with habitat characteristics to estimate abundance and distribution in an endangered cryptic bird. Biodivers Conserv 26:1525-1539

Ellner SP, Fieberg J, Ludwig D, Wilcox C (2002) Precision of population viability analysis. Conserv Biol 16:258-261

Ely CA, Clapp RB (1973) The natural history of Laysan Island, Northwestern Hawaiian Islands. Atoll Res Bull No. 171

Fantle-Lepczyk J, Taylor A, Duffy DC, Crampton LH, Conant S (2016) Weather influences on nest success of the endangered puaiohi (Myadestes palmeri). Wilson $\mathrm{J}$ Ornithol 128:43-55

Fantle-Lepczyk JE, Taylor A, Duffy DC, Crampton LH, Conant S (2018) Using population viability analysis to evaluate management activities for an endangered Hawaiian endemic, the puaiohi (Myadestes palmeri). PLOS ONE 13:e0198952

Fortini LB, Vorsino AE, Amidon FA, Paxton EH, Jacobi JD (2015) Large-scale range collapse of Hawaiian forest birds under climate change and the need for 21st century conservation options. PLOS ONE 10:e0140389

Freifeld H, Plentovich S, Farmer C, Kohley CR and others (2016) Long-distance translocations to create a second millerbird population and reduce extinction risk. Biol Conserv 199:146-156

Gilpin ME, Soulé ME (1986) Minimum viable populations: the processes of species extinction. In: Soulé ME (ed) Conservation biology: the science of scarcity and diversity. Sinauer, Sunderland, MA, p 13-34

Griffith B, Scott JM, Carpenter JW, Reed C (1989) Translocation as a species conservation tool: status and strategy. Science 245:477-480

Groom MJ, Pascual MA (1998) The analysis of population persistence: an outlook on the practice of viability analysis. In: Fiedler PL, Kareiva PM (eds) Conservation biology, 2nd edn. Chapman and Hall, New York, NY, p 4-27

*Hamilton S, Moller H (1995) Can PVA models using com- puter packages offer useful conservation advice? Sooty shearwaters (Puffinus griseus) in New Zealand as a case study. Biol Conserv 73:107-117

Hawai'i Department of Land and Natural Resources (2016) Breeding program for rare Hawaiian bird closes, 18 puaiohi released. http://dlnr.hawaii.gov/blog/2016/04/ 01/nr16-064/ (accessed on 9 July 2016)

James HF, Olson SL (1991) Descriptions of thirty-two new species of birds from the Hawaiian Islands: Part II: Passeriformes. Ornithol Monogr 46:1-88

Kepler CB, Kepler AK (1983) A first record of the nest and chicks of the small Kauai thrush. Condor 85:497-499

Kilpatrick AM (2006) Facilitating the evolution of resistance to avian malaria in Hawaiian birds. Biol Conserv 128: 475-485

Komdeur J (1997) Inter-island transfers and population dynamics of Seychelles warblers, Acrocephalus sechellensis. Bird Conserv Int 7:7-26

Lacy RC (2000) Considering threat to the viability of small populations using individual-based models. Ecol Bull 48: 39-51

Lacy RC, Pollak JP (2014) Vortex: a stochastic simulation of the extinction process (version 10.0). Chicago Zoological Society, Brookfield, IL

* Lindenmayer DB, Possingham HP (1996) Ranking conservation and timber management options for Leadbeater's possum in southeastern Australia using population viability analysis. Conserv Biol 10:235-251

Ludwig D (1996) Uncertainty and the assessment of extinction probabilities. Ecol Appl 6:1067-1076

Meine CD, Archibald GW (1996) The cranes - status survey and conservation action plan. International Union for Conservation of Nature and Natural Resources, Gland and Cambridge

*Meretsky VJ, Snyder NFR, Beissinger SR, Clendenen DA, Wiley JW (2000) Demography of the California condor: implications for reestablishment. Conserv Biol 14: 957-967

Morris WF, Doak DF (2002) Quantitative conservation biology: theory and practice of population viability analysis. Sinauer Associates, Sunderland, MA

Owen-Smith N (2007) Introduction to modeling in wildlife and resource conservation. Blackwell Publishing, Malden, MA

Perkins R (1903) Vertebrata. In: Sharp D (ed) Fauna Hawaiiensis, Vol 1, part IV. University Press, Cambridge

Pratt TK (2009) Origins and evolution. In: Pratt TK, Atkinson CT, Banko PC, Jacobi JD, Woodworth BL (eds) Conservation biology of Hawaiian forest birds: implications for island avifauna. Yale University Press, New Haven, CT, p 3-24

Richardson F, Bowles J (1964) A survey of the birds of Kauai, Hawaii. B. P. Bishop Museum Bulletin No. 227. Bishop Museum Press, Honolulu, HI.

Scott JM, Mountainspring S, Ramsey FL, Kepler CB (1986) Forest bird communities of the Hawaiian Islands: their dynamics, ecology, and conservation. Stud Avian Biol 9: $1-431$

Scott JM, Conant S, Van Riper C III (2001) Introduction to evolution, ecology, and management of Hawaiian birds: a vanishing avifauna. Stud Avian Biol 22:1-12

Shaffer ML (1981) Minimum population sizes for species conservation. Bioscience 31:131-134

Snetsinger TJ, Wakelee KM, Fancy SG (1999) Puaiohi (Myadestes palmeri). In: Poole AF, Gill FB (eds) The 
birds of North America, No. 461. The Birds of North America, Philadelphia, PA

Snetsinger TJ, Herrmann CM, Holmes DE, Hayward CD, Fancy SG (2005) Breeding ecology of the puaiohi (Myadestes palmeri). Wilson Bull 117:72-84

Switzer R, Lieberman A, Nelson J, Crampton LH (2013) Augmentation of the puaiohi population through captive propagation and release on the Alakai Plateau, Kauai, Hawaii, USA. Reintroduction Specialist Group Book. International Union for Conservation of Nature and Natural Resources, Gland and Cambridge

Taylor BL (1995) The reliability of using population viability analysis for risk classification of species. Conserv Biol 9: 551-558

Tweed EJ, Foster JT, Woodworth BL, Monahan WB, Kellerman JL, Lieberman A (2006) Breeding biology and success of a reintroduced population of the critically endangered puaiohi. Auk 123:753-763

USFWS (US Fish and Wildlife Service) (2006) Puaiohi, Myadestes palmeri. In: Revised recovery plan for Hawaiian forest birds. US Fish and Wildlife Service, Region 1, Portland, OR, p 2.31-2.46

Editorial responsibility: J. Michael Reed, Medford, Massachusetts, USA
VanderWerf EA (2009) Importance of nest predation by alien rodents and avian poxvirus in conservation of Oahu elepaio. J Wildl Manag 73:737-746

VanderWerf EA, Mosher SM, Burt MD, Taylor PE, Sailer D (2011) Variable efficacy of rat control in conserving Oahu elepaio populations. In: Veitch CR, Clout MN, Towns DR (eds) Island invasives: eradication and management. International Union for Conservation of Nature and Natural Resources, Gland and Cambridge, p 124-130

* VanderWerf EA, Crampton LH, Diegmann JS, Atkinson CT, Leonard DL (2014) Survival estimates of wild and captive-released puaiohi, an endangered Hawaiian thrush. Condor 116:609-618

*Wilcove DS, Rothstein D, Dubow J, Phillips A, Losos E (1998) Quantifying threats to imperiled species in the United States. Bioscience 48:607-615

Woodworth BL, Pratt TK (2009) Life history and demography. In: Pratt TK, Atkinson CT, Banko PC, Jacobi JD, Woodworth BL (eds) Conservation biology of Hawaiian forest birds: implications for island avifauna. Yale University Press, New Haven, CT, p 194-233

Submitted: March 1, 2019; Accepted: November 14, 2019 Proofs received from author(s): January 24, 2020 